\title{
A Proposed Framework for the Implementation of Early Infant Diagnosis Point-of-Care
}

\author{
Karidia Diallo, Surbhi Modi, Mackenzie Hurlston, ${ }^{1}$ R. Suzanne Beard, ${ }^{1}$ and John N. Nkengasong ${ }^{1}$
}

\begin{abstract}
Early diagnosis of HIV infection in infants and children remains a challenge in resource-limited settings, with approximately half of all HIV-exposed infants receiving virological testing for HIV by the recommended age of 2 months in 2015. To reduce morbidity and mortality among HIV-infected children and close the treatment gap for HIV-infected children, there is an urgent need to evaluate existing programmatic and laboratory practices for early infant diagnosis and introduce strategies to improve identification of HIV-exposed infants and ensure access to systematic, early HIV testing, with early linkage to treatment for HIV-infected infants. This article describes progress made in follow-up of HIV-exposed infants since 2006, including remaining unmet laboratory and programmatic needs, and recommends strategies for improvement, especially those related to the implementation of point-of-care technology for early infant diagnosis.
\end{abstract}

Keywords: HIV, point-of-care, early infant diagnosis, near point-of-care

\section{Introduction}

$\mathbf{I}^{\mathrm{N}}$ N 2015, 1.4 MILLION CHILDREN were estimated to be living with HIV, with the vast majority living in sub-Saharan Africa. ${ }^{1}$ As prevention of mother-to-child HIV transmission (PMTCT) programs have expanded, the number of children infected with HIV annually has declined by $50 \%$ since 2010 ; however, 150,000 children were still newly infected with HIV in $2015^{1}$ and 150,000 children died due to HIV-related causes in 2014. ${ }^{2}$ Without antiretroviral treatment (ART), more than half of HIV-infected children die by the age of two. ${ }^{3-8}$ Despite the clear need for early treatment, only $49 \%$ of children living with HIV received ART in 2015, which falls short of global treatment targets. ${ }^{1}$

The global public health community has called for rapid action through policy and program initiatives designed to increase treatment access for children living with HIV, including the U.S. President's Emergency Plan for AIDS Relief (PEPFAR) partnership with the Children's Investment Fund Foundation (CIFF) for the Accelerating Children's HIV/ AIDS Treatment (ACT) Initiative and World Health Organization (WHO) guidelines recommending universal ART eligibility for all children. These initiatives highlight the need to refocus attention on improving identification of children living with HIV, including a special focus on improving earlier identification of HIV-infected infants who are at the highest risk of mortality. This article reviews programmatic gaps in follow-up of HIV-exposed infants along with progress with conventional systems for early infant diagnosis and provides recommendations for the introduction of point-ofcare (POC) technologies to facilitate early diagnosis of HIV among infants.

\section{Identification and Follow-Up of HIV-Exposed Infants: Progress and Challenges}

Access to timely HIV diagnosis for HIV-exposed infants is a critical step to close the treatment coverage gap for children and reduce HIV-associated mortality for children. Because maternal HIV antibodies persist in HIV-exposed infants up to the age of 18 months, early HIV diagnosis in infants requires the use of virological testing. Although virological testing is offered at or near the time of birth to high-risk infants in the United States, WHO has previously recommended testing HIV-exposed infants in resource-limited settings using polymerase chain reaction (PCR) technology at age 4-6

\footnotetext{
${ }^{1}$ International Laboratory Branch and ${ }^{2}$ Maternal and Child Health Branch, Division of Global HIV and Tuberculosis, Centers for Disease Control and Prevention, Atlanta, Georgia.

(C) Karidia Diallo, et al., 2017; Published by Mary Ann Liebert, Inc. This Open Access article is distributed under the terms of the Creative Commons Attribution Noncommercial License (http://creativecommons.org/licenses/by-nc/4.0/) which permits any noncommercial use, distribution, and reproduction in any medium, provided the original author(s) and the source are credited.
} 
weeks. ${ }^{9,10}$ This time point has been recommended to allow alignment with routine healthcare visits for immunization services and optimize detection of intrauterine, intrapartum, and early postnatal transmissions. However, despite substantial investments in building capacity for early infant diagnosis, only $42 \%$ of HIV-exposed infants received a virological test for HIV within the first 2 months of life in 2014 . $^{2}$ In 2015 , only 4 of the 21 countries in sub-Saharan Africa prioritized for PMTCT as part of the Global Plan toward the elimination of new HIV infections among children by 2015 and keeping their mothers alive (the Global Plan) and provided virological testing to more than half of HIVexposed infants in the first 2 months of life; three countries provided testing to less than $10 \%$ of HIV-exposed infants. ${ }^{1}$ Multiple factors contribute to the low rates of early testing of HIV-exposed infants, including the lack of diversified testing and/or sample collection sites (i.e., HIV testing or sample collection is mainly offered at PMTCT sites), stock outs of HIV testing commodities at the facility and central laboratory levels, poorly functioning sample transport networks, and delays and gaps in returning results to caregivers. ${ }^{4,11-14}$ Nuwagaba-Biribonwoha et al. showed how the lapses in return of results to caregivers strongly limited children's access to early infant diagnosis care and treatment in Tanzania. ${ }^{15}$ Delays, at times up to several months for a caregiver to receive test results, is a critical issue given the high mortality for HIV-infected infants that are not detected and treated early. ${ }^{16}$ For those who survive, delays in treatment initiation due to delays in early HIV diagnosis contribute to poor clinical outcomes. ${ }^{17-19}$

The clinical continuum of care for HIV-exposed infants begins with identification and treatment of HIV-infected pregnant and/or breastfeeding women and requires systematic follow-up and determination of final HIV status through the end of the breastfeeding period, with linkage to treatment for HIV-infected infants. In 2015, 77\% of pregnant women living with HIV worldwide had access to antiretroviral medicines to prevent transmission of HIV to their infants. ${ }^{1}$ While this represents substantial success in PMTCT programs, this falls short of the coverage recommended as part of virtual elimination of mother-to-child HIV transmission goals and represents gaps along the cascade of HIV-related services for pregnant and breastfeeding women. In many resource-limited settings, pregnant women choose to deliver at home or present for antenatal care late in pregnancy, thus missing opportunities for HIV testing and treatment initiation in early pregnancy and increasing the risk of mother-to-child transmission. Systematic programs for repeat HIV testing of pregnant women who test negative at their first antenatal visit need to be strengthened through delivery and breastfeeding to ensure identification of women with incident HIV infections and identification of all HIV-exposed infants.

As countries have expanded Option B+ (or "Treat All") programs for PMTCT, increasing numbers of women are receiving ART for life. This offers the promise of protection against HIV transmission for future pregnancies; however, multiple countries have reported high rates of loss to followup for pregnant and breastfeeding women in Option B+ programs. $^{20,21}$ Innovative strategies are being implemented to improve retention of mothers and their infants, including the use of mobile technology for patient appointment reminders and return of results from reference laboratories to clinical facilities, and enhanced engagement of communities through targeted interventions and peer-supported groups. ${ }^{22-24}$ PMTCT programs in multiple countries including Kenya, Mozambique, and Malawi have implemented monitoring and evaluation tools, including birth cohort registers and linked identifiers for mothers and infants, to facilitate longitudinal tracking of mother-infant pairs through the end of breastfeeding period ${ }^{25}$

Although most efforts to improve identification and follow-up of HIV-exposed infants have been located within antenatal clinic and PMTCT settings, targeted efforts are needed to reach women and their infants at other venues. For example, integrating systematic HIV testing services for women and infants into immunization clinics and other highyield settings for pediatric HIV case finding, such as TB clinics, malnutrition clinics, and inpatient wards, offers an opportunity for identifying HIV-infected women and HIVexposed and HIV-infected infants who are missed within the PMTCT program. To expand and integrate services outside of PMTCT settings, programs must evaluate and plan to meet human resource, training, and commodity needs.

\section{Access to Early Infant Diagnosis Testing: Progress and Challenges}

An important step in the expansion of early infant HIV testing services in resource-limited settings occurred at the WHO-PEPFAR meeting in Uganda in $2006,{ }^{26}$ where dried blood spots (DBS) were designated as the preferred specimen type and DNA PCR as the recommended testing method for early infant diagnosis. ${ }^{27}$ From 2006 to 2009, PEPFAR supported early infant diagnosis expansion in $>25$ resourcelimited countries by developing laboratory capacity for DNA PCR testing, providing proficiency testing panel free of charge for laboratory quality testing assessment, and training of laboratorians for early infant diagnosis PCR testing. ${ }^{28}$ With combined support from Ministries of Health, PEPFAR, and numerous other partners and donors, the use of DBS for early infant diagnosis has expanded and the number of laboratories performing early infant diagnosis testing has increased from 17 laboratories in 11 countries in 2006 to more than 136 laboratories in 41 countries by the end of 2012. ${ }^{19,29-36}$

Although use of DBS specimens has facilitated sample collection and transportation, early infant diagnosis requires sophisticated nucleic acid testing, typically through highthroughput technology that is expensive, ${ }^{1,33,37}$ and also highly trained staff and highly functional laboratory infrastructure, ${ }^{26}$ which are generally present only in large, centralized laboratories (Fig. 1). Requirements for highly qualified laboratory personnel are a major barrier in resourcelimited settings, where there is less than one laboratory professional for 10,000 people. ${ }^{38}$ Laboratory systems in subSaharan Africa also face a high turnover rate as there are often unsatisfactory working conditions and requirements for public service rotations within rural regions for laboratorians, negatively affecting both the quality and access to early infant HIV testing. ${ }^{39,40}$

In many countries, delays in processing and communicating results, particularly in returning results from the central laboratory to the clinic and from the clinic to caregivers, limit access to early infant diagnosis. ${ }^{41-43}$ However, there have historically been limited alternatives to conventional 
FIG. 1. Early infant diagnosis testing pyramid stratified by tiered laboratory level, infrastructure, and staff capacity.

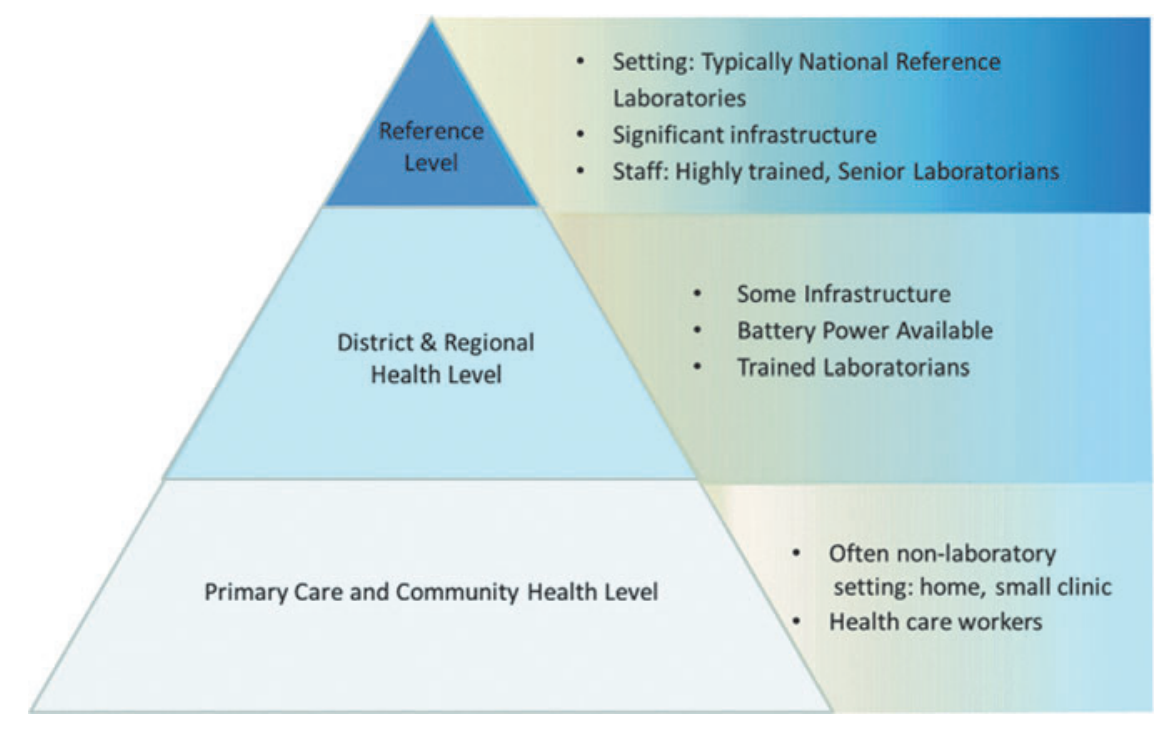

centralized reference laboratory systems for virological testing in resource-limited settings. Manual PCR assays, both laboratory developed and commercial, can be performed at smaller sites, but these methods are quite time-consuming and require highly skilled personnel to ensure quality testing. Most district-, regional-, and primary-level clinics were not suited for these early infant diagnosis PCR assays and had no alternative but to send specimens to the central laboratory for testing (Fig. 1).

\section{Role of POC Technologies}

POC technology may have the potential for expanding infant HIV virological testing to lower tiers throughout the laboratory network. Given the persistent challenges in sample transport and results return systems for early infant diagnosis, POC technologies that would allow for provision of results to caregivers on the same day as specimen collection offer new hope for reaching the United Nations Programme on HIV and AIDS (UNAIDS) 90-90-90 targets (by 2020, 90\% of all people living with HIV will know their HIV status; $90 \%$ of all people with diagnosed HIV infection will receive sustained antiretroviral therapy; and $90 \%$ of all people receiving antiretroviral therapy will have viral suppression) for HIV-exposed infants. In June 2016, WHO prequalified the first two POC qualitative assays to be used for early infant diagnosis of HIV: the Xpert ${ }^{\circledR}$ HIV-1 Qual Assay for use on the GeneXpert Systems and the Alere ${ }^{\mathrm{TM}} \mathrm{q}$ HIV-1/2 Detect. Both assays are easy to use, require minimal operator intervention, and produce a result rapidly (50 min on Alere q HIV1/2 Detect; 90 min on Xpert HIV-1 Qual), although the Xpert HIV-1 Qual Assay is a near POC technology (i.e., intended for use in laboratories where electricity is accessible, cannot be operated at primary healthcare settings with no electricity). For optimal usage and the greatest impact on patient care, these new instruments will require proper placement within the existing efficient laboratory network as well as coordinated healthcare systems to ensure effective treatment and true linkage to pediatric care for HIV-positive infants. In the next section, we describe the laboratory and program con- siderations that will be needed and recommend some key strategies to achieve the full promise of this technology.

\section{Recommended evaluations before introduction of POC technologies for early infant diagnosis}

POC technology offers an appealing solution for scaling up early infant diagnosis testing as they tend to be small, userfriendly instruments that can be operated with minimal training by nonlaboratory staff and in clinical service delivery settings with the potential to provide accurate results within a few hours. While POC technology offers the opportunity for countries to diversify early infant diagnosis networks and scale-up early infant diagnosis both quickly and efficiently, a clearly defined roadmap to determine POC utility and suitability should be developed and implemented before implementation of POC use in routine diagnosis of HIV.

We propose a roadmap that includes the following phasedapproach in evaluating and introducing POC for early infant diagnosis: phase 1 laboratory-based evaluation, phase 2 fieldbased evaluation, and phase 3 implementation evaluation. This roadmap involves a series of thorough evaluations completed independent of the manufacturers, on products that are considered past the experimental/research-only phase of development. Independent laboratory and field evaluations cannot be replaced by Conformité Européenne (CE) marking or Expert Review Panel for Diagnostics (EPRD), which serve as indications for procurement for products sold in the European Economic Area ${ }^{44}$ and Global Fund purchasing, ${ }^{45}$ respectively.

Phase 1 laboratory-based evaluations are designed to confirm the manufacturer's claim of sensitivity and specificity, as well as limit of detection, reproducibility, and operational requirements. This phase of evaluation is completed in a laboratory with experience in technology and assay evaluations. The laboratories used for the laboratory evaluation are preferably certified to International Standard Organization (ISO) or an equivalent standard. The results of this phase can be utilized for WHO prequalification, as has been done for Alere q HIV-1/2 Detect and Xpert HIV-1 Qual. 
Ideally, the laboratory-based evaluation phase should be preferably completed before phase 2 , the field implementation evaluations. Field evaluations are generally completed in locations of intended use. During the field evaluation phase, the results obtained from the POC device may still need to be confirmed using standard-of-care virological testing procedures in use in the country performing the evaluation. However, because of the high mortality associated with early HIV infection, countries may choose to start ART on the same day as receipt of POC diagnoses, while obtaining a DBS sample for testing using the conventional early infant diagnosis platform. However, each country may need to make this decision based on local context and turnaround times (TAT) for conventional testing. The field evaluation (phase 2 evaluation) provides valuable information about how the POC device stands up under routine use in adverse conditions as well as how the intended users utilize the testing platform. Training needs for intended users can be thoroughly vetted at this stage. Error rates are likely to be different when intended users perform the assays, and this information is tracked routinely to determine if the error rates are due to use by a specific user, inadequate training, or device issues. Quality assurance (QA) and quality control (QC) are implemented allowing for requirements to be adjusted to fit the needs and standards of quality necessary for use in diagnosis.

Phase 3 involves implementation evaluation, which should occur after the field evaluation has been completed or as the field evaluation is concluding. This final phase of evaluation is key for a country to conduct itself to ensure that the POC device will function as described in their particular testing environment and cascade. At this point, the POC device is ready to be utilized for diagnosis and this phase of evaluation will help guide the country in developing a plan on when, where, and how to implement POC early infant diagnosis within their specific infant HIV testing algorithm. Implementation studies will help determine if the test alleviates some of the issues that are inherent with high-throughput early infant diagnosis testing and specimen transport issues and will also help determine best placement within the system for the POC devices. At a minimum, data could be gathered on number of patients tested and started on ART, TAT, cost of testing, acceptance, and feasibility versus the conventional testing method.

When all evaluations have been completed, before countrywide rollout and purchasing of POC technology to improve early infant diagnosis testing, it will be important to address issues such as the availability of POC devices and reduction of time for prequalification and regulatory approval. Special consideration would also be needed for determination of appropriate placement and integration within the healthcare system. Data gathered from the laboratory, field, and implementation evaluations will assist with these decisions. These considerations will be described in the following section.

\section{Determining when and where to implement POC technologies for early infant diagnosis}

To maximize the potential benefits of POC infant HIV testing, it is important to understand the lessons learned from implementation of other POC technologies, including HIV serology, CD4 POC (e.g., Pima), malaria rapid testing, on- site testing for maternal syphilis, and Xpert MTB/Rif testing for tuberculosis. ${ }^{14,46-50}$ Analysis of Pima placement and utilization data highlighted inefficiencies related to poor placement of machines; $34 \%$ of the sites with a Pima device used it $\leq 1$ time per day, while $30 \%$ of the sites where Pima was implemented had easy access to a referral laboratory and did not need a POC device. ${ }^{51}$ Also, despite reducing time to tuberculosis diagnosis, Xpert/Rif implementation did not reduce mortality at 6 months compared with sputum microscopy, leading to the observation that improving outcomes in drug-sensitive tuberculosis programs might require not only more rapid diagnostic tests but also better linkage to care. ${ }^{52}$ Other studies have demonstrated the benefit of POC technology, including showing that Pima enabled clinics to stage patients rapidly onsite after enrolment, improving assessment for ART eligibility and reducing opportunities for pretreatment loss to follow-up. ${ }^{47,53}$ As a result, more patients were identified as eligible for and were initiated on ART, supporting the fact that CD4 POC could be an effective intervention to reduce pretreatment loss to follow-up.

When planning for the introduction of POC testing for infant HIV testing, it is important that countries view this technology as an extension of the conventional laboratory network for early infant diagnosis rather than a substitute for or competition with a fully functional laboratory system. Even with the introduction of POC devices, systems for monitoring and evaluation, proficiency testing, and external QA of early infant diagnosis testing will rely on linkages with conventional laboratory networks. Most POC testing can be performed by all cadres of healthcare staff with appropriate training, but task sharing to reduce the additional work must be acceptable and adequately planned for within the country, including ensuring that all cadres of workers who will utilize POC technology will receive in-depth training. They will also need to be encouraged to have functional and ongoing collaborations with the laboratories at the district and central levels where QA and QC for POC testing should be implemented and coordinated. Collaboration across laboratory levels for training, as well as monitoring of results for QA purposes, is essential to ensure quality early infant diagnosis testing.

Addition of POC testing into any early infant diagnosis cascade will also require determining how to incorporate this technology into the country's current testing algorithm. Countries will need to determine whether the POC test result will function as a stand-alone result or whether confirmation will be required from another blood sample, including whether samples should be tested with the same POC device or with another type of test. While WHO recommends performing a confirmatory HIV test by another tester with another sample before a patient is given ART, ${ }^{54}$ a POC virological test result might be very well suited as a first test to initiate treatment for an infant or child to minimize the risk of loss-to-follow up and HIV-related mortality while waiting for a confirmatory test either from another blood draw test on the same POC device or tested by other means such as conventional PCR.

Considerable planning of existing early infant diagnosis and laboratory infrastructure is also needed to define where POC devices will be best utilized. Mapping the needs of each country may be performed to ensure that each POC device is placed appropriately as these devices may fill several different niches depending on the country and existing 
FIG. 2. Building an efficient early infant diagnosis laboratory network within existing infrastructure.
A
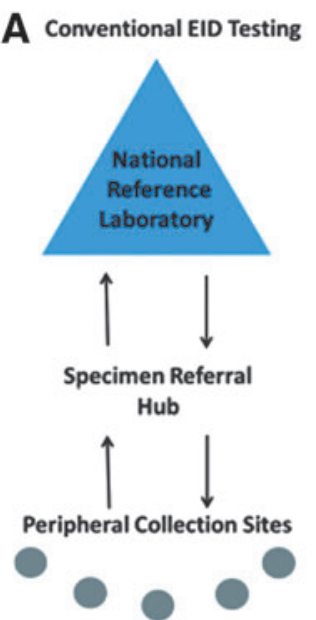
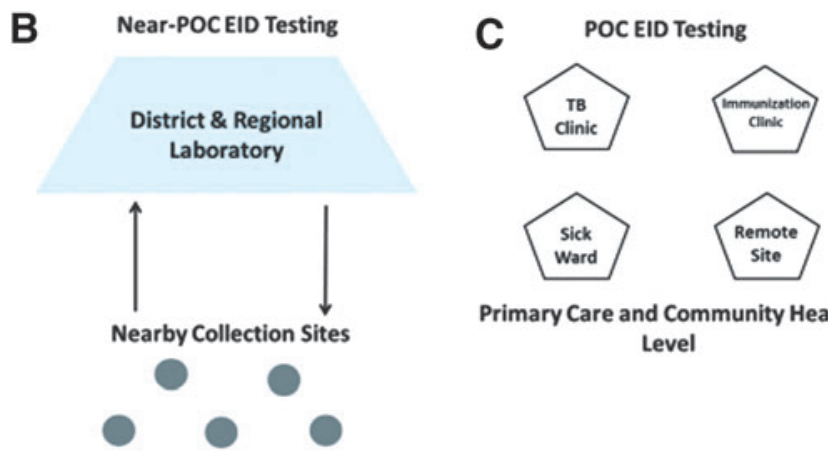

Primary Care and Community Health Level

Infrastructure Requirements

laboratory networks. Figure 2 A shows how peripheral sites close to a hub can send their specimens to the hub, which then sends them to the level III high-throughput laboratory. Figure 2B shows how a testing site serving nearby collection sites with a substantial number of specimens (a district or regional hospital/health center) might need more than one POC device for an efficient testing TAT, which might not be an efficient usage of a POC instrument. Placement of a nearPOC device, such as Xpert, in a district or regional laboratory will allow nearby collection sites to use that instrument efficiently. The placement of multiple POC instruments in a testing site to meet the site's testing needs should be avoided as much as possible for a more efficient usage of each device. Nearby specimen referral hub systems have been shown to efficiently receive specimens from several sites with low daily or weekly specimens' number and transport them to regional, provincial, or reference laboratories. ${ }^{55}$ The reverse process (reference to regional or provincial laboratories to hub to peripheral sites) can then be used for results return. Other strategies for results return include the use of short message service (SMS) printers and other cell phone technologies, which can make the result return process even more efficient. ${ }^{56}$ For example, use of the volunteer quality corps (Q-corps) officers (a concept modeled on the U.S. Peace Corps program used to support and improve community projects in various departments, including health, agriculture, commerce, and education) has significantly reduced the TAT from 30 to 5 days for Cameroon HIV diagnosis QA testing program. ${ }^{57}$

Overall, POC testing may be particularly important for early identification of HIV-infected infants and children in remote settings, at immunization clinics, and other targeted intervention sites (Fig. 2C), and may facilitate early linkage to ART, which may ultimately be life-saving for severely ill infants and children. Providing virological testing at or near the sick child, including in the inpatient pediatric wards, tuberculosis clinics, programs for malnourished children, immunization services, maternity wards, adult HIV clinics, and/ or any others departments within the health facility where HIV-exposed and/or sick infants may be found, may significantly increase access to early infant HIV diagnosis. Testing at sites where sick children receive services may allow for more rapid results return to the physician or nurse in charge of the child's health management and may facilitate more rapid caregiver notification and prompt treatment initiation. However, performing early infant diagnosis testing outside of the laboratory will require additional planning, training, coordination, and monitoring to be successful. There may not be one perfect setting for POC testing, and for high-volume sites, POC devices will most likely not meet testing demands.

Even with POC testing, effective systems for linkage to early ART are key; the ultimate measure of the impact of POC devices will be whether their use translates into earlier initiation of ART and reduced infant mortality due to HIV. To achieve this outcome, clear systems for communication between all the individuals involved in the continuum of care for HIV-exposed and HIV-infected infants will still be required. Expanding access to early infant HIV testing supports multiple global health goals, including efforts to achieve the UNAIDS 90-90-90 targets for children and the call for universal health coverage. ${ }^{58}$

\section{Conclusion}

POC infant HIV testing offers many opportunities, especially in a context of universal health coverage; however, to maximize their full potential for facilitating early linkage to HIV care and treatment, early infant diagnosis POC must not be seen as a stand-alone solution and must instead be properly integrated into laboratory and clinical networks. Today, two early infant diagnosis POC that are CE-marked have been thoroughly and independently evaluated and approved by WHO prequalification program. As countries gear up for their usage and the introduction of additional technologies, the following components of planning will be critical: (1) planning, communication, and coordination between all entities involved in the care and treatment cascade of children; (2) training of healthcare workers and laboratorians on all aspects of early infant HIV diagnosis, including new POC technologies; (3) integration of POC devices within the existing laboratory network; (4) implementation of a strong QA and QC program for POC devices; and (5) monitoring and evaluation to allow implementation of improvement processes. 
Following the steps outlined above will help ensure that each level of the clinical and laboratory system is covered (either by specimen transport, POC infant HIV testing, or conventional virological testing) and will facilitate the greatest access, coverage, and impact of this critical service. POC testing may close the gaps within the early infant diagnosis testing and treatment cascade, and link additional infants to care and treatment, however, improving existing laboratory network infrastructure will be important as well. For early infant diagnosis POC to positively impact pediatric care and treatment, these technologies cannot be implemented without careful assessment and strengthening of the entire clinical and laboratory cascade.

\section{Acknowledgment}

This report has been supported by the PEPFAR through the Centers for Disease Control and Prevention (CDC).

\section{Disclaimer}

The opinion expressed in this review article are those of the authors and do not necessarily represent the official position of the Centers for Disease Control and Prevention/the Agency for Toxic Substances and Disease Registry.

\section{Author Disclosure Statement}

No competing financial interests exist.

\section{References}

1. UNAIDS: On the fast-track to an AIDS-free generation: The incredible journey of the Global Plan towards the elimination of new HIV infections among children by 2015 and keeping their mothers alive. Available at www.unaids .org/sites/default/files/media_asset/GlobalPlan2016_en.pdf (2016), accessed July 10, 2016.

2. UNAIDS: How AIDS Changed Every Thing. MDG 6: 15 Years, 15 Lessons of Hope from the AIDS response. Geneva 2015.

3. Dunn D, Woodburn P, Duong T, et al.: Current CD4 cell count and the short-term risk of AIDS and death before the availability of effective antiretroviral therapy in HIVinfected children and adults. J Infect Dis 2008;197:398-404.

4. Ghadrshenas A, Ben Amor Y, Chang J, et al.: Improved access to early infant diagnosis is a critical part of a childcentric prevention of mother-to-child transmission agenda. AIDS 2013;27 Suppl 2:S197-S205.

5. Marston M, Becquet R, Zaba B, et al.: Net survival of perinatally and postnatally HIV-infected children: A pooled analysis of individual data from sub-Saharan Africa. Int $\mathbf{J}$ Epidemiol 2011;40:385-396.

6. Munyagwa M, Baisley K, Levin J, Brian M, Grosskurth H, Maher D: Mortality of HIV-infected and uninfected children in a longitudinal cohort in rural south-west Uganda during 8 years of follow-up. Trop Med Int Health 2012;17:836-843.

7. Newell ML, Brahmbhatt H, Ghys PD: Child mortality and HIV infection in Africa: A review. AIDS 2004;18 Suppl 2: S27-S34.

8. Newell ML, Coovadia H, Cortina-Borja M, et al:: Mortality of infected and uninfected infants born to HIV-infected mothers in Africa: A pooled analysis. Lancet 2004;364:1236-1243.

9. HHS Panel: Guidelines for the use of antiretroviral agents in pediatric HIV infection. Available at https://aidsinfo.nih
.gov/contentfiles/lvguidelines/PediatricGuidelines.pdf (2015), accessed July 10, 2016.

10. WHO: WHO Recommendations on the diagnosis of HIV infection in infants and children. Available at http:// whqlibdoc.who.int/publications/2010/9789241599085_eng .pdf?ua=1, accessed July 10, 2016.

11. Ciaranello AL, Park JE, Ramirez-Avila L, Freedberg KA, Walensky RP, Leroy V: Early infant HIV-1 diagnosis programs in resource-limited settings: Opportunities for improved outcomes and more cost-effective interventions. BMC Med 2011;9:59.

12. Penazzato M, Revill P, Prendergast AJ, et al.: Early infant diagnosis of HIV infection in low-income and middleincome countries: Does one size fit all? Lancet Infect Dis 2014;14:650-655.

13. Sherman GG, Stevens G, Jones SA, Horsfield P, Stevens WS: Dried blood spots improve access to HIV diagnosis and care for infants in low-resource settings. J Acquir Immune Defic Syndr 2005;38:615-617.

14. Wolpaw BJ, Mathews C, Chopra M, et al.: The failure of routine rapid HIV testing: A case study of improving low sensitivity in the field. BMC Health Serv Res 2010;10:73.

15. Nuwagaba-Biribonwoha $\mathrm{H}$, Werq-Semo B, Abdallah A, et al.: Introducing a multi-site program for early diagnosis of HIV infection among HIV-exposed infants in Tanzania. BMC Pediatr 2010;10:44.

16. Wagner A, Slyker J, Langat A, et al.: High mortality in HIV-infected children diagnosed in hospital underscores need for faster diagnostic turnaround time in prevention of mother-to-child transmission of HIV (PMTCT) programs. BMC Pediatr 2015;15:10.

17. Braun M, Kabue MM, McCollum ED, et al: Inadequate coordination of maternal and infant HIV services detrimentally affects early infant diagnosis outcomes in Lilongwe, Malawi. J Acquir Immune Defic Syndr 2011;56:e122-e128.

18. Chatterjee A, Tripathi S, Gass $\mathrm{R}$, et al:: Implementing services for Early Infant Diagnosis (EID) of HIV: A comparative descriptive analysis of national programs in four countries. BMC Public Health 2011;11:553.

19. Hassan AS, Sakwa EM, Nabwera HM, et al.: Dynamics and constraints of early infant diagnosis of HIV infection in Rural Kenya. AIDS Behav 2012;16:5-12.

20. Domercant JW, Puttkammer N, Lu LS, Francois K, Duncan D, Grand'Pierre R, Lowrance D, Adler M. Attrition from antiretroviral treatment services among pregnant and nonpregnant patients following adoption of Option B+ in Haiti IAS Vancouver, 2015.

21. Tenthani L, Haas AD, Tweya $\mathrm{H}$, et al.: Retention in care under universal antiretroviral therapy for HIV-infected pregnant and breastfeeding women ('Option B+') in Malawi. AIDS 2014;28:589-598.

22. Schmitz K, Scheepers E, Okonji E, Kawooya V: Retaining mother-baby-pairs in care and treatment: The mothers2mothers Mentor Mother Model. Available at www.m2m .org/wp-content/uploads/2015/07/IAS2015_KathrinSchmitz Presentation.pdf, accessed July 10, 2016.

23. Kieffer MP, Woelk G, Mpofu D, Cathcart R; the ACCLAIM Study Team: Improving early ANC attendance through community engagement and dialogue: Project ACCLAIM in three African countries. Available at https:// www.m2m.org/wp-content/uploads/2015/07/IAS2015_ KathrinSchmitzPresentation.pdf, accessed July 10, 2016.

24. Mwapasa V, Pro G, Chinkhumba J, et al.: Mother-infant pair clinic and SMS messaging as innovative strategies for 
improving access to and retention in eMTCT care and Option B+ in Malawi: A cluster randomized control trial (the PRIME study). J Acquir Immune Defic Syndr 2014;67 Suppl 2:S120-S124.

25. Inter-Agency Task Team on the Prevention and Treatment of HIV Infection in Pregnant Women, Mother and Children: $\mathrm{B}+$ monitoring \& evaluation framework dissemination and country consultation. Adapting monitoring \& evaluation systems for cohort and enhanced monitoring as well as outcome and impact evaluations/assessments. IATT, October 20-23, 2015. Available at http://emtct-iatt .org/wp-content/uploads/2016/04/Executive-Summary_Final_ 22April2016.pdf, accessed July 10, 2016.

26. Stevens W, Sherman G, Downing R, et al: Role of the laboratory in ensuring global access to ARV treatment for HIV-infected children: Consensus statement on the performance of laboratory assays for early infant diagnosis. Open AIDS J 2008;2:17-25.

27. Ou CY, Yang H, Balinandi S, et al.: Identification of HIV-1 infected infants and young children using real-time RT PCR and dried blood spots from Uganda and Cameroon. J Virol Methods 2007;144:109-114.

28. International Laboratory Branch: CDC is establishing and strengthening international laboratories for rapid effective disease response. Available at www.cdc.gov/globalaids/ publications/laboratory_factsheet.pdf (2012), accessed July 10, 2016.

29. Garcia A, Subbarao S, Zhang G, et al:: Impact of proficiency testing program for laboratories conducting early diagnosis of HIV-1 infection in infants in low- to middleincome countries. J Clin Microbiol 2014;52:773-780.

30. Audu R, Onwuamah C, Salu O, et al.: Development and implementation challenges of a quality assured HIV infant diagnosis program in Nigeria using dried blood spots and DNA polymerase chain reaction. AIDS Res Hum Retroviruses 2015;31:433-438.

31. Chiduo MG, Mmbando BP, Theilgaard ZP, et al.: Early infant diagnosis of HIV in three regions in Tanzania; successes and challenges. BMC Public Health 2013;13:910.

32. Creek T, Tanuri A, Smith M, et al.: Early diagnosis of human immunodeficiency virus in infants using polymerase chain reaction on dried blood spots in Botswana's national program for prevention of mother-to-child transmission. Pediatr Infect Dis J 2008;27:22-26.

33. Creek TL, Sherman GG, Nkengasong J, et al.: Infant human immunodeficiency virus diagnosis in resource-limited settings: Issues, technologies, and country experiences. Am J Obstet Gynecol 2007;197(3 Suppl):S64-S71.

34. Nkenfou CN, Lobe EE, Ouwe-Missi-Oukem-Boyer O, et al.: Implementation of HIV early infant diagnosis and HIV type 1 RNA viral load determination on dried blood spots in Cameroon: Challenges and propositions. AIDS Res Hum Retroviruses 2012;28:176-181.

35. Noubiap JJ, Bongoe A, Demanou SA: Mother-to-child transmission of HIV: Findings from an early infant diagnosis program in Bertoua, Eastern Cameroon. Pan Afr Med J 2013; 15:65.

36. Torpey K, Mandala J, Kasonde P, et al.: Analysis of HIV early infant diagnosis data to estimate rates of perinatal HIV transmission in Zambia. PLoS One 2012; 7:e42859.

37. Sherman GG, Matsebula TC, Jones SA: Is early HIV testing of infants in poorly resourced prevention of mother to child transmission programmes unaffordable? Trop Med Int Health 2005;10:1108-1113.
38. Tsehaynesh P: ASLM: Building laboratory capacity in Africa in a sustainable way. Int J Lab Hematol 2014;36:18.

39. Fitzgibbon JE, Wallis CL: Laboratory challenges conducting international clinical research in resource-limited settings. J Acquir Immune Defic Syndr 2014;65 Suppl 1: S36-S39.

40. Marinucci F, Majigo M, Wattleworth M, Paterniti AD, Hossain MB, Redfield R: Factors affecting job satisfaction and retention of medical laboratory professionals in seven countries of Sub-Saharan Africa. Hum Resour Health 2013; 11:38.

41. Deo S, Crea L, Quevedo J, et al.: Expedited results delivery systems using GPRS technology significantly reduce early infant diagnosis test turnaround times. J Acquir Immune Defic Syndr 2015;70:e1-4.

42. Mugambi ML, Deo S, Kekitiinwa A, Kiyaga C, Singer ME: Do diagnosis delays impact receipt of test results? Evidence from the HIV early infant diagnosis program in Uganda. PLoS One 2013;8:e78891.

43. Sutcliffe CG, van Dijk JH, Hamangaba F, Mayani F, Moss WJ: Turnaround time for early infant HIV diagnosis in rural Zambia: A chart review. PLoS One 2014;9:e87028.

44. CE marking-European Commission. Available at https:// ec.europa.eu/growth/single-Market/ce-marking_en (2015), accessed July 10, 2016.

45. The Global Fund, UNITAID: Quality assurance policy for diagnostics. The expert review panel mechanism. Available at www.theglobalfund.org/.../PSM_QualityAssurancePolicy Diagnostics_Presentation_en (2014), accessed July 10, 2016.

46. Jani IV, Peter TF: How point-of-care testing could drive innovation in global health. N Engl J Med 2013;368:2319-2324.

47. Jani IV, Sitoe NE, Alfai ER, et al.: Effect of point-of-care CD4 cell count tests on retention of patients and rates of antiretroviral therapy initiation in primary health clinics: An observational cohort study. Lancet 2011;378:1572-1579.

48. Mkwanazi NB, Patel D, Newell ML, et al.: Rapid testing may not improve uptake of HIV testing and same day results in a rural South African community: A cohort study of 12,000 women. PLoS One 2008;3:e3501.

49. Myer L, Wilkinson D, Lombard C, Zuma K, Rotchford K, Karim SS: Impact of on-site testing for maternal syphilis on treatment delays, treatment rates, and perinatal mortality in rural South Africa: A randomised controlled trial. Sex Transm Infect 2003;79:208-213.

50. Theron G, Zijenah L, Chanda D, et al.: Feasibility, accuracy, and clinical effect of point-of-care Xpert MTB/RIF testing for tuberculosis in primary-care settings in Africa: A multicentre, randomised, controlled trial. Lancet 2014;383:424-435.

51. Jason W: Perspective on current CD4 POC deployment and implementation; considerations for EID POC. EID POCT Workshop, Atlanta, November 20, 2014. Abstract 20A.

52. Churchyard GJ, Stevens WS, Mametja LD, McCarthy KM, Chihota V, Nicol MP, Erasmus LK, Ndjeka NO, Mvusi L, Vassall A, Sinanovic E, Cox HS, Dye C, Grant AD, Fielding KL:. Xpert MTB/RIF versus sputum microscopy as the initial diagnostic test for tuberculosis: A clusterrandomised trial embedded in South African roll-out of Xpert MTB/RIF. Lancet Global Health 2015;3:e450-e457.

53. Patten GE, Wilkinson L, Conradie K, et al.: Impact on ART initiation of point-of-care CD4 testing at HIV diagnosis among HIV-positive youth in Khayelitsha, South Africa. J Int AIDS Soc 2013;16:18518.

54. WHO: WHO reminds national programmes to retest all newly diagnosed people with HIV. Available at www.who 
.int/hiv/pub/vct/retest-newly-diagnosed-plhiv-full/en (2014), accessed July 10, 2016.

55. Kiyaga $\mathrm{C}$, Sendagire H, Joseph E, et al.: Uganda's new national laboratory sample transport system: A successful model for improving access to diagnostic services for Early Infant HIV Diagnosis and other programs. PLoS One 2013;8:e78609.

56. WHO/CHAI: SMS printers aid early infant diagnosis of HIV-ADS in Nigeria. CHAI's SMART. Available at http:// apps.who.int/iris/bitstream/10665/92801/1/WHO_RHR_13.19_ eng.pdf (2013), accessed July 10, 2016.

57. Ndazi Juliana P: The Use of Quality Corps Volunteers and Quality Assurance of HIV Testing. Paper presented at ASLM, December 3-8, 2014. Cape Town, SA.

58. The World Bank: 500+ organizations launch global coalition to accelerate access to universal health coverage.
Available at www.worldbank.org/en/news/press-release/ 2014/12/12/500-organizations-global-coalition-accelerateaccess-universal-health-coverage, accessed July 10, 2016.

Address correspondence to: Karidia Diallo International Laboratory Branch, Mail Stop G-45 Division of Global HIV and Tuberculosis Centers for Disease Control and Prevention 1600 Clifton Road, NE Atlanta, GA 30333

E-mail: edu9@cdc.gov 